\title{
The requisite for acid generation to solubilize insoluble salts may account for surge of glucose levels in some diabetes patients
}

Lipeng Liao, Yuhan Lin, Chanyuk Lam, Jess Lan Ouyang, Min Fu, Man Tang, Qiuyun Liu

School of Life Sciences, Sun Yat-sen University, Guangzhou 510275, China.

$\triangle$ Correspondence: Qiuyun Liu, Ph.D, School of Life Sciences, Sun Yat-sen University, Guangzhou 510275, China. Tel: $\left[\begin{array}{llll}+86 & (020) & 84110296\end{array}\right]$ (lsslqy@mail.sysu.edu.cn). 
Abstarct

Diabetes is a comorbidity of COVID-19, and the mortality may be pertinent to the wide distribution of insoluble and stiff salts such as calcium oxalate in diabetic sufferers. As an intrinsic self-protective mechanism, tissues in diabetic individuals may build up hydrogen-bonding-capable molecules such as glucose for the generation of acids which can solubilize insoluble and stiff salts or crystals. 
A study demonstrated that fasting blood glucose at hospital admission was significantly associated with 28-day mortality in patients with COVID-19 without prior diagnosis of diabetes [1]. Diabetes is a common comorbidity of COVID-19 [2], and the mortality may be related to the broad distribution of insoluble and rigid salts such as stressful calcium oxalate in diabetic sufferers. As an innate self-protective cellular machinery, tissues in diabetic individuals may build up hydrogen-bonding-capable molecules such as glucose for the production of acids which can solubilize waterinsoluble and stiff salts or crystals [3], in a way somewhat similar to heating a solution to increase solubility of some insoluble and rigid substances.

The proteins of SARS-CoV-2 virus are high in valine plus glycine which have long carbonyl bond lengths and are projected to possess potent secondary chemical bonding and bind calcium effectively [3-6]. Oxalate can be generated from Krebs cycle, gluconeogenesis and other pathways [3], particular in winter when energy metabolism is robust. Calcium oxalate is the primary component of renal stones and detrimental to cells and tissues. Kidney stones can lead to renal failure and shorten lifespan in animals [7]. The compounding of the problem concerning insoluble and stiff salts in diabetic sufferers with viral infections can worsen the condition resulting in serious consequences. The gastrointestinal manifestations in a modest fraction of the diabetic sufferers suggest that insoluble and rigid salts were present [8]. A study found that COVID-19 patients with Type 2 diabetes had a high mortality rate of $7.8 \%$ (adjusted hazard ratio, 1.49) [9]. Various measures such as starch-based diet, fasting plus boiled rice water, fasting plus orange juice, etc., can be adopted to address the needs of COVID-19 patients during the ongoing pandemic besides vaccination $[3,4,10]$, as these measures can restrict calorie or amino acid intake and attenuate energy metabolism. The approach was documented in mediaeval times as "starving the fever", and tried and tested in history in China and in western countries.

\section{Acknowledgement}

This work was supported by grant from Guangzhou Science and Technology Program (201804010328) to Q. Liu. We thank Yan Shi for editing. 


\section{References:}

1. Wang S, Ma P, Zhang S, Song S, Wang Z, Ma Y, et al. Fasting blood glucose at admission is an independent predictor for 28-day mortality in patients with COVID19 without previous diagnosis of diabetes: a multi-centre retrospective study. Diabetologia. 2020; 63(10):2102-2111. https://doi.org/10.1007/s00125-020-052091

2. Muniyappa R, Gubbi S. COVID-19 pandemic, coronaviruses, and diabetes mellitus. Am J Physiol Endocrinol Metab. 2020;318(5):E736-E741. https://doi.org/10.1152/ajpendo.00124.2020

3. Wan Y, Yan S, Zhang Y, An S, Yang K, Xu H, Gan T, et al. The Pneumonia outbreak: High isoleucine and high valine plus glycine contents are features of the proteins of COVID-19 virus. Preprints. 2020. doi: 10.20944/preprints202002.0289.v5

4. Yan S, Zhang Y, Liu Q. Why COVID-19 virus is so deadly to cancer patients? Eur J Cancer Prev. 2020;29(4):365. https://doi.org/10.1097/CEJ.0000000000000605

5. Zhang X, Ma X, Gan T, Shi Y, Wang Y, Liu Q. Secondary Chemical Bonding between Insoluble Calcium Oxalate and Carbonyl Oxygen Atoms of GLY and VAL Residues Triggers the Formation of A $\beta$ Aggregates and Their Deposition in the Brain. ACS Chem Neurosci. 2020;11(24):400711.https://doi.org/10.1021/acschemneuro.0c00662

6. Wang Y, Tang JW, Ma WQ, Feng J, Feng J. Dietary zinc glycine chelate on growth performance, tissue mineral concentrations, and serum enzyme activity in weanling piglets. Biol Trace Elem Res. 2010;133(3):325-34. https://doi.org/10.1007/s12011009-8437-3

7. Hall JA, Yerramilli M, Obare E, Li J, Yerramilli M, Jewell DE. Serum concentrations of symmetric dimethylarginine and creatinine in cats with kidney stones. PloS one. $2017 ; 12(4)$ : e0174854. https://doi.org/10.1371/journal.pone.0174854

8. Sommers, T., Mitsuhashi, S., Singh, P., Hirsch, W., Katon, J., Ballou, et al. Prevalence of Chronic Constipation and Chronic Diarrhea in Diabetic Individuals 
in the United States. Am J Gastroenterol. 2019;114(1), 135-142. https://doi.org/10.1038/s41395-018-0418-8

9. Zhu L, She ZG, Cheng X, Qin JJ, Zhang XJ, Cai J, et al. Association of Blood Glucose Control and Outcomes in Patients with COVID-19 and Pre-existing Type 2 Diabetes. Cell Metab. 2020;31(6):1068-1077.e3. https://doi.org/10.1016/j.cmet.2020.04.021

10. Gan T, Fu M, Wu J, Wen L, Liu Q. How to design carbohydrate diet regimens for heart disease patients. Eur J Prev Cardiol. 2018;25(9):979-980. https://doi.org/10.1177/2047487318766602 